\title{
Reflets
}

Revue ontaroise d'intervention sociale et communautaire

\section{Les défis du travail de développement communautaire auprès de la communauté somalienne de la Basse-Ville d'Ottawa}

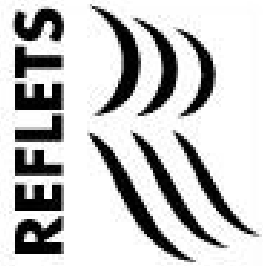

\section{Richard Monette}

Volume 4, numéro 1, printemps 1998

Intervention en contextes minoritaires

URI : https://id.erudit.org/iderudit/026207ar

DOI : https://doi.org/10.7202/026207ar

Aller au sommaire du numéro

Éditeur(s)

Reflets : Revue ontaroise d'intervention sociale et communautaire

ISSN

1203-4576 (imprimé)

1712-8498 (numérique)

Découvrir la revue

Citer cet article

Monette, R. (1998). Les défis du travail de développement communautaire auprès de la communauté somalienne de la Basse-Ville d'Ottawa. Reflets, 4(1), 194-199. https://doi.org/10.7202/026207ar

Tous droits réservés (C) Reflets : Revue ontaroise d'intervention sociale et communautaire, 1998
Ce document est protégé par la loi sur le droit d'auteur. L'utilisation des services d'Érudit (y compris la reproduction) est assujettie à sa politique d'utilisation que vous pouvez consulter en ligne.

https://apropos.erudit.org/fr/usagers/politique-dutilisation/ 


\section{Les défis du travail de dévelop- pement communautaire auprès de la communauté somalienne de la Basse-Ville d'Ottawa}

\section{Richard Monette}

Agent de développement communautaire, Centre de ressources de la Basse-Ville d'Ottawa.

Le quartier de la Basse-Ville d'Ottawa a connu deux vagues de changements majeurs depuis vingt-cinq ans. Dans un premier temps, ce milieu résidentiel a été transformé radicalement par un programme de rénovation urbaine au cours des années 1970. Quelques années plus tard, c'est-à-dire au début des années 1990, plusieurs centaines de familles issues de minorités raciales se sont établies dans le milieu et ce, principalement dans les logements subventionnés. Près de mille unités de logement social ont été construites en guise de remplacement des centaines de maisons qui ont disparu lors de la démolition des années 1970.

\section{Les changements intervenus dans la Basse-Ville d'Ottawa}

Pour sa part, le Centre de ressources de la Basse-Ville (CRBV) a ouvert ses portes au mois d'octobre 1970, dans le but de planifier l'amélioration des services destinés à la population principalement 
francophone et durement touchée par le programme de rénovation urbaine. Vingt-sept années plus tard, le CRBV offre plusieurs services à la population locale, dont l'intervention en situation de crise et le counselling auprès des familles et des enfants ainsi que la formation de groupes et d'activités communautaires, tout en appuyant les groupes et organismes du milieu et les actions collectives. Notons cependant que ces services sont maintenant offerts, entre autres, à une population francophone minoritaire (moins de $40 \%$ ) et à une population immigrante grandissante (environ $20 \%$ ). Plusieurs des nouveaux arrivants sont originaires des pays suivants : la Somalie, la Chine, l'Iran, la Roumanie, Haïti et le Liban. La plupart de ces nouveaux résidants ont un faible revenu et s'adaptent plus ou moins bien à leur nouveau milieu. Ce sont cependant les soixante familles somaliennes qui manifestent le plus de besoins, à cause de problèmes liés à la pauvreté et à l'accès aux services sociaux.

Le personnel du Centre de ressources de la Basse-Ville tâche depuis maintenant trois ans de travailler conjointement avec des représentants de la communauté somalienne dans le but de réduire les obstacles de culture et de langue qui nuisent à l'accès aux services publics, aux emplois ainsi qu'aux services de soutien personnel.

L'adaptation au milieu de la Basse-Ville, qui s'avère très longue pour la population somalienne, est des plus évidentes au sein des familles où le rôle des membres a beaucoup changé. Contrairement à ce qu'il en était en Somalie, les femmes n'ont plus de réseaux d'entraide et se retrouvent souvent très isolées. Elles ont, pour la plupart, cinq enfants et dans certains cas, jusqu'à huit ou neuf. Peu d'hommes détiennent des emplois et les seuls revenus proviennent souvent de l'assistance sociale.

Les jeunes, quant à eux, surtout ceux âgés de moins de 10 ans, se sont adaptés plus rapidement à la société canadienne. Cette situation a créé des conflits entre parents et enfants et au sein même des couples. Par la pratique sociale qu'est le développement communautaire, nous visons donc à combattre les inégalités économiques et la discrimination vécue et à favoriser l'accès aux services et programmes gouvernementaux. Nous nous adressons aux minorités raciales en général et à la communauté somalienne 
en particulier afin de favoriser leur auto-développement. Nous regroupons cette population en fonction de divers contextes, soit dans leurs quartiers, en tant que communautés d'intérêt (problèmes sociaux spécifiques, absence d'emploi, etc.) et en tant que communauté d'identité (groupe ethnique).

\section{L'identification des besoins}

Avant de discuter des modes d'intervention utilisés et des défis à relever, il est de mise de décrire les besoins et les moyens utilisés pour les identifier.Premièrement, contractuels, stagiaires et personnel permanent du CRBV ont tous et toutes rencontré divers membres de la communauté somalienne et ont pu identifier ses besoins par l'intermédiaire de cafés-rencontres, de groupes de discussion, de cours de cuisine et d'habiletés parentales. Deuxièmement, un comité formé de quatre membres dits représentatifs de la communauté somalienne nous a fait part régulièrement de ses souhaits et de ses doléances. Enfin, dans un troisième temps, nous avons effectué un porte à porte systématique auprès de soixante familles du quartier afin de sonder leurs besoins. Cette dernière tâche a été réalisée par deux intervenants d'origine somalienne.

Nous pouvons alors regrouper les besoins prioritaires de la façon suivante: besoins immédiats tout d'abord: nourriture, logement, sécurité; connaissance des services disponibles; mécanismes pour briser l'isolement; services de soutien et d'appui personnel; intégration aux activités et organismes du milieu et finalement, formation et accès au marché du travail.

\section{Les modes d'intervention}

Pour faire face aux besoins, nous privilégions deux formes d'intervention présentant chacune des défis de différente 
envergure. Au niveau individuel, nous répondons aux besoins des femmes en matière de services particuliers tels l'alimentation (dépannage, cours de cuisine et groupe de cuisine collective), le logement (représentation auprès des pourvoyeurs en matière de litiges), la violence conjugale (counselling et référence aux services) et le soutien personnel (enfants, isolement, accompagnement dans les cas reliés à l'immigration).

Ces interventions ont surtout comme but l'élimination des barrières linguistiques et culturelles. Grâce aux contractuelles et aux stagiaires d'origine somalienne et à l'interprétation culturelle, nous arrivons à livrer ces services avec passablement de succès. D'autre part, ces interventions se font dans un contexte d'entraide, de coopération et d'encouragement à la prise en charge personnelle. Nous devons aussi être conscients que nos propres valeurs et préjugés ont un impact sur notre façon d'intervenir et en tenir compte quotidiennement.

La deuxième forme d'intervention se fait en collaboration avec des membres de la communauté somalienne dans le but d'accroître les possibilités d'emploi et d'offrir des services de soutien. Il s'agit d'élaborer des mécanismes qui permettront à cette collectivité de s'adapter et d'intégrer le marché du travail. Ensemble, nous avons décidé d'entreprendre diverses activités afin d'aider les gens aptes au travail à se préparer pour obtenir et conserver un emploi ou un travail indépendant. Voici quelquesunes de ces activités : recherche de financement aux fins d'achat de formation auprès d'agences gouvernementales et de fondations; services d'aide à l'emploi, c'est-à-dire aide au diagnostique et développement des compétences en recherche d'emploi; counselling et activités connexes à l'endroit des personnes qui éprouvent des difficultés à se trouver de l'emploi (estime de soi, etc.); identification de stages de travail pratiques chez des employeurs afin de donner une expérience canadienne aux personnes qui sont ouvertes à l'exploration de nouvelles avenues et de nouveaux domaines d'occupation.

Nous voulons donc améliorer la qualité de vie des membres de la communauté somalienne car nous visons à surmonter les obstacles, à la fois personnels et structurels, à la vie autonome. Pour ce faire, 
les intervenants et intervenantes du Centre de ressource de la BasseVille cherchent l'apport des membres élus du comité somalien.

\section{Les défis de l'intervention}

C'est à ce stade que les défis sont nombreux car pour que le processus en soit un d'autodétermination, il faut une implication réelle et concrète des membres. Dans le dossier de la formation et de l'emploi, la participation est trop souvent modeste. Selon les personnes impliquées dans le projet, cette participation modeste s'explique de diverses façons. En Somalie, le rôle du gouvernement et des instances publiques est très limité dans le domaine de l'emploi, qui relève du secteur privé. Dans ce cas, la recherche d'emploi se fait de façon informelle et à travers des contacts personnels. Une fois au Canada, il devient donc parfois difficile de s'impliquer dans des processus d'élaboration de projets d'employabilité, bureaucratiques et impersonnels, qui peuvent prendre plusieurs mois, surtout si l'on attend des subventions et des approbations ministérielles. L'impatience devant des processus en apparence abstraits et qui ne mèneront à l'emploi qu'à moyen terme, nuirait donc à une plus grande collaboration. Afin de réussir, notre approche communautaire bénéficierait donc surtout de pouvoir aider directement à trouver de vrais emplois à court terme. Nous savons cependant que les emplois ne sont pas toujours disponibles et ce, indépendamment des barrières culturelles. La mise sur pied de sessions de formation en emploi est loin d'être suffisante et ne répond pas aux attentes de personnes dont l'emploi est nécessaire à la survie et à la dignité.

D'autre part, certaines lacunes évidentes se dressent en barrières devant cette population désireuse d'intégrer le marché du travail. La discrimination à l'embauche, bien sûr, mais aussi le manque de services de garde, qui sont très limités au niveau de l'accès (places subventionnées) ou trop dispendieux. De plus, comme nous l'avons $\mathrm{vu}$, les réseaux d'entraide ne sont pas encore en place dans notre quartier, en particulier pour les femmes. 
À la suite de nos interventions, nous croyons qu'il est beaucoup plus facile d'offrir des services qui ne requièrent qu'une participation immédiate, par exemple, des groupes d'exercice ou de natation, des cours de cuisine ou de couture. Par contre, quand il s'agit d'élaborer et de mettre sur pied des activités répondant «sur mesure» aux besoins des participants potentiels, nous demandons aux gens de participer à des comités, d'être co-auteurs de projets, d'être cosignataires de demandes de subvention.

Nous constatons alors que la participation communautaire s'effrite. En tant qu'intervenants communautaires, nous devons alors faire face à une contradiction qui fait tout le défi de la démarche d'empowerment communautaire. Lorsque nous tentons d'impliquer les gens dans le développement des services et que nous mettons l'accent sur leurs forces, leurs talents et leurs habiletés, nous nous butons à une sorte de résistance qui est sans doute causée par l'incompréhension de l'importance que nous attribuons à cette démarche. Nous tentons bien d'expliquer le processus d'empowerment que nous visons et nous insistons sur la nécessité de ne pas créer de dépendance, car le financement n'est que ponctuel et a pour but d'aider à développer des idées. Cependant, cette explication ne semble pas suffisante.

Face à ce constat, nous avons choisi de créer un groupe de réflexion rassemblant des intervenants d'origine somalienne et des intervenants communautaires d'autres centres de ressources, travaillant auprès de la communauté somalienne d'Ottawa. Notre but est de partager nos expériences afin de parvenir à une stratégie commune plus régionale que locale en matière d'emploi. Nous devons également insister sur l'importance de miser sur des services de soutien pour les jeunes afin que ces derniers puissent se frayer un chemin dans notre société compétitive et se trouver des emplois. Dans ce domaine, nous avons à ce jour rassemblé suffisamment de parents qui s'engageront auprès de bénévoles chargés d'offrir un programme d'études supervisées après les classes afin d'améliorer l'apprentissage scolaire. Avant tout, cependant, nous tentons de répondre à des besoins sociaux et nous travaillons à l'organisation démocratique d'un milieu. 